PROCEEDINGS OF THE

AMERICAN MATHEMATICAL SOCIETY

Volume 131, Number 1, Pages 55-63

S 0002-9939(02)06508-5

Article electronically published on May 8, 2002

\title{
LINEAR OPERATORS PRESERVING CORRELATION MATRICES
}

\author{
CHI-KWONG LI AND STEVE PIERCE
}

(Communicated by Joseph A. Ball)

\begin{abstract}
The linear operators that map the set of real or complex (rank one) correlation matrices onto itself are characterized.
\end{abstract}

\section{INTRODUCTION}

Let $M_{n}(\mathbf{F})$ be the set of $n \times n$ matrices over $\mathbf{F}=\mathbf{R}$ or $\mathbf{C}$. A matrix in $M_{n}(\mathbf{F})$ is a correlation matrix if it is positive semi-definite with all diagonal entries equal to one. Correlation matrices arise in the study of statistics as well as many other pure and applied areas. Instead of giving a long list of references, we suggest the readers do a keyword search on the subject in MathSciNet to see the many related papers.

Let $C_{n}(\mathbf{F})$ be the set of correlation matrices in $M_{n}(\mathbf{F})$ and let $V_{n}(\mathbf{F})$ be the real linear space spanned by $C_{n}(\mathbf{F})$. One readily checks that $V_{n}(\mathbf{R})$ (respectively, $V_{n}(\mathbf{C})$ ) is the set of $n \times n$ real symmetric (respectively, complex Hermitian) matrices with constant diagonal entries. The purpose of this note is to characterize those real linear operators on $V_{n}(\mathbf{F})$ mapping $C_{n}(\mathbf{F})$ onto itself. We always assume that $n \geq 2$, to avoid trivial considerations.

A generalized permutation matrix is a square matrix of the form $D P$, where $P$ is a permutation matrix and $D$ is a diagonal matrix satisfying $D^{*} D=I$. Clearly, if $P$ is a generalized permutation matrix and $\phi$ is a mapping on $V_{n}(\mathbf{F})$ defined by $A \mapsto P^{*} A P$ or $A \mapsto P^{*} A^{t} P$ when $\mathbf{F}=\mathbf{C}$, then $\phi\left(C_{n}(\mathbf{F})\right)=C_{n}(\mathbf{F})$. It turns out that the converse is also valid. Actually, we only need to assume that $\phi$ maps the set of rank one correlation matrices onto itself to conclude that $\phi$ is of the above forms. More precisely, we have the following result.

Theorem 1.1. Let $\phi$ be a linear operator on $V_{n}(\mathbf{F})$. The following conditions are equivalent.

(a) $\phi\left(C_{n}(\mathbf{F})\right)=C_{n}(\mathbf{F})$.

(b) $\phi(\mathcal{R})=\mathcal{R}$, where $\mathcal{R}$ is the set of rank one correlation matrices.

(c) There is a generalized permutation matrix $P$ such that (c.i) $\phi(A)=P^{*} A P$ for all $A \in V_{n}(\mathbf{F})$, or

(c.ii) $\mathbf{F}=\mathbf{C}$ and $\phi(A)=P^{*} A^{t} P$ for all $A \in V_{n}(\mathbf{F})$.

Received by the editors October 17, 2000 and, in revised form, August 18, 2001.

2000 Mathematics Subject Classification. Primary 15A04.

Key words and phrases. Correlation matrix, linear operator.

Research supported by an NSF grant. 
We remark that characterizing linear operators $\phi$ on $V_{n}(\mathbf{F})$ satisfying $\phi\left(C_{n}(\mathbf{F})\right) \subseteq$ $C_{n}(\mathbf{F})$ is a more difficult problem, and there are many low rank linear operators satisfying this property. For example, any mapping of the form $A \mapsto(\operatorname{tr} A) B / n$ for a fixed correlation matrix $B$ is such a map. Also, one can adapt the mapping of Choi [2] on $3 \times 3$ matrices to $V_{3}(\mathbf{F})$ defined by

$$
\left(\begin{array}{lll}
a_{11} & a_{12} & a_{13} \\
a_{21} & a_{22} & a_{23} \\
a_{31} & a_{32} & a_{33}
\end{array}\right) \mapsto \frac{1}{2}\left(\begin{array}{ccc}
2 a_{11} & -a_{12} & -a_{13} \\
-a_{21} & 2 a_{22} & -a_{23} \\
-a_{31} & -a_{32} & 2 a_{33}
\end{array}\right) .
$$

One readily checks that this mapping is invertible and maps $C_{n}(\mathbf{F})$ into itself, but it is not onto. For instance, the matrix

$$
\frac{1}{3}\left(\begin{array}{lll}
3 & 2 & 2 \\
2 & 3 & 2 \\
2 & 2 & 3
\end{array}\right) \in V_{3}(\mathbf{F})
$$

but its preimage is not a correlation matrix. Hence, this map is not of the form described in Theorem 1.1. In fact, the study of linear operators mapping $C_{n}(\mathbf{F})$ into itself is related to the very hard problem of characterizing positive linear maps; see [5, Chapter 3].

In our discussion, denote by $\left\{e_{1}, \ldots, e_{n}\right\}$ the standard basis for $\mathbf{R}^{n}$. Let $e=$ $\sum_{j=1}^{n} e_{j}, E=e e^{t}$, and $E_{i j}=e_{i} e_{j}^{t}$ for $1 \leq i, j \leq n$. The usual inner product on square matrices induces a (real) inner product on $V_{n}(\mathbf{F})$ defined by $(A, B)=$ $\operatorname{tr}\left(A B^{*}\right)=\operatorname{tr}(A B)$. We present some auxiliary results in the next section, and the proof of the main theorem in the last section.

\section{Auxiliary Results}

To prove our main theorem we establish some auxiliary results which are of independent interest.

Proposition 2.1. Let $G$ be a compact group of invertible linear operators $\phi$ on $V_{n}(\mathbf{F})$ containing the group $G_{0}$ of linear operators of the form $A \mapsto P^{*} A P$, where $P$ is a generalized permutation matrix. Then there exists a linear operator $\xi$ of the form $A \mapsto \alpha(\operatorname{tr} A) I+A$, where $\alpha>-1 / n$, such that $\xi G \xi^{-1}$ is a subgroup of the group of orthogonal operators on $V_{n}(\mathbf{F})$. Consequently, each linear operator in $G$ will preserve the inner product $(A, B)_{\xi}=(\xi(A), \xi(B))$ on $V_{n}(\mathbf{F})$.

Proof. Let $G$ satisfy the hypotheses of the proposition. By the result in [1] (see also [4]), there exists a positive definite linear operator $\xi$ on $V_{n}(\mathbf{F})$ such that $\xi G \xi^{-1}$ is a subgroup of the group of orthogonal operators on $V_{n}(\mathbf{F})$.

For any generalized permutation matrix $P$, let $\psi_{P}$ be the operator $A \mapsto P^{*} A P$. Since $\psi_{P} \in G$, we see that $\xi \circ \psi_{P} \circ \xi^{-1}$ is an orthogonal operator, i.e., the operator $\left(\xi \psi_{P} \xi^{-1}\right)^{*}\left(\xi \psi_{P} \xi^{-1}\right)$ is the identity. As $\psi_{P}^{*}=\psi_{P}^{-1}$, we have

$$
\left(\xi^{*} \xi\right) \psi_{P}=\psi_{P}\left(\xi^{*} \xi\right)
$$

Observe that the group $G_{0}$ of operators $\psi_{P}$, where $P$ is a generalized permutation matrix, has two irreducible subspaces in $V_{n}(\mathbf{F})$, namely, (i) the span of $I$, and (ii) the subspace of matrices in $V_{n}(\mathbf{F})$ with zero diagonal. Let $\mathcal{B}=\left\{I_{n} / \sqrt{n}, B_{2}, \ldots, B_{m}\right\}$ be an orthonormal basis for $V_{n}(\mathbf{F})$; here $m=\operatorname{dim} V_{n}(\mathbf{F})$. Then the projection of 
$A \in V_{n}(\mathbf{F})$ on $\operatorname{span}\left\{B_{2}, \ldots, B_{m}\right\}$ is exactly $A-(\operatorname{tr} A / n) I$. Suppose the operator matrix of $\xi^{2}$ with respect to $\mathcal{B}$ is

$$
M=\left(\begin{array}{cc}
\gamma & v^{*} \\
v & H
\end{array}\right) .
$$

We are going to show that $v=0$ and $H=\beta I_{m-1}$ for some $\beta>0$. If $V_{n}(\mathbf{F})=$ $V_{2}(\mathbf{R})$, then there are two operators in $G_{0}$, whose operator matrices with respect to $\mathcal{B}$ are $I_{2}$ and $E_{11}-E_{22}$. Since $M$ commutes with them, $M$ is of the asserted form. If $V_{n}(\mathbf{F}) \neq V_{2}(\mathbf{R})$, then the two subspaces are of different dimensions. By Schur's lemma (e.g., see [3, p.182]), we get the desired conclusion that $v=0$ and $H=\beta I_{m-1}$.

Since $\xi$ is positive definite and $\xi^{*} \xi=\xi^{2}$, it follows that $\xi$ also acts as scalar operators on these subspaces. Thus, there exist positive numbers $\mu, \eta$ such that

$$
\xi(A)=\mu \frac{\operatorname{tr} A}{n} I+\eta\left(A-\frac{\operatorname{tr} A}{n} I\right) .
$$

We may replace $\xi$ by $\xi / \eta$ and assume that $\eta=1$. Let $\alpha=(\mu-1) / n>-1 / n$. Then $\xi$ is of the form $A \mapsto \alpha(\operatorname{tr} A) I+A$, as asserted.

Since $\xi \phi \xi^{-1}$ is orthogonal, we have

$$
\begin{aligned}
(\phi(A), \phi(B))_{\xi} & =(\xi \phi(A), \xi \phi(B)) \\
& =\left(\xi \phi \xi^{-1} \xi(A), \xi \phi \xi^{-1} \xi(B)\right) \\
& =(\xi(A), \xi(B)) \\
& =(A, B)_{\xi}
\end{aligned}
$$

The result follows.

By Proposition 2.1 we have the following corollary.

Corollary 2.2. Let $G$ be the set of linear operators $\phi$ on $V_{n}(\mathbf{F})$ satisfying $\phi\left(C_{n}(\mathbf{F})\right)$ $=C_{n}(\mathbf{F})$ or $\phi(\mathcal{R})=\mathcal{R}$. Then $G$ is a compact group satisfying the hypotheses and hence the conclusion of Proposition 2.1.

Proof. One can easily check that both $C_{n}(\mathbf{F})$ and $\mathcal{R}$ are compact sets spanning $V_{n}(\mathbf{F})$. Thus, the set of invertible operators mapping any of these sets onto itself is a compact group. Clearly, the group contains $G_{0}$ as a subgroup. The result follows.

Proposition 2.3. Let $\xi$ be defined as in Proposition 2.1 For any $A \in C_{n}(\mathbf{F})$ we have

$$
n+2 \alpha n^{2}+\alpha^{2} n^{3} \leq(A, A)_{\xi} \leq n^{2}+2 \alpha n^{2}+\alpha^{2} n^{3} .
$$

The first equality holds if and only if $A=I$; the second equality holds if and only if A has rank one.

Proof. Note that

$$
(A, A)_{\xi}=(A+\alpha n I, A+\alpha n I)=(A, A)+2 \alpha n^{2}+\alpha^{2} n^{3} .
$$

Suppose $A$ has eigenvalues $\lambda_{1} \geq \cdots \geq \lambda_{n} \geq 0$. Then $\lambda_{1}+\cdots+\lambda_{n}=n$ and $(A, A)=\lambda_{1}^{2}+\cdots+\lambda_{n}^{2}$. By elementary calculus, one sees that

$$
n \leq \lambda_{1}^{2}+\cdots+\lambda_{n}^{2} \leq n^{2} .
$$

The first equality holds if and only if $\lambda_{1}=\cdots=\lambda_{n}$; the second equality holds if and only if $\lambda_{1}=n$ and $\lambda_{2}=\cdots=\lambda_{n}=0$. The result follows. 
Proposition 2.4. If $\phi$ is a linear operator on $V_{n}(\mathbf{F})$ satisfying $\phi\left(C_{n}(\mathbf{F})\right)=C_{n}(\mathbf{F})$, then $\phi(\mathcal{R})=\mathcal{R}$.

Proof. Let $\phi$ satisfy the hypothesis of the proposition. By Corollary 2.2

$$
(\phi(A), \phi(B))_{\xi}=(A, B)_{\xi} .
$$

If $A \in \mathcal{R}$, then

$$
(\phi(A), \phi(A))_{\xi}=(A, A)_{\xi}=n^{2}+2 \alpha n^{2}+\alpha^{2} n^{3} .
$$

By Proposition 2.3, $\phi(A)$ is rank one. So, $\phi(\mathcal{R}) \subseteq \mathcal{R}$. Applying the arguments to $\phi^{-1}$, we see that $\phi^{-1}(\mathcal{R}) \subseteq \mathcal{R}$. The result follows.

Proposition 2.5. If $\phi$ is a linear operator on $V_{n}(\mathbf{F})$ mapping $\mathcal{R}$ onto itself, then the following conditions hold.

(1) $\phi(I)=I$.

(2) $\phi$ preserves the inner product on $V_{n}(\mathbf{F})$, i.e.,

$$
(\phi(A), \phi(B))=(A, B) \quad \text { for any } A, B \in V_{n}(\mathbf{F}) .
$$

Proof. Suppose $\phi$ is a linear map on $V_{n}(\mathbf{F})$ such that $\phi(\mathcal{R})=\mathcal{R}$. Let $\mathcal{S}$ be the convex hull of $\mathcal{R}$. Then $\phi(\mathcal{S})=\mathcal{S}$. Note that $E=e e^{t} \in \mathcal{R}$, and

$$
I=\frac{1}{2^{n}}\left\{\sum_{j=1}^{2^{n}} D_{j} E D_{j}\right\} \in \mathcal{S},
$$

where $D_{1}, \ldots, D_{2^{n}}$ are all the $n \times n$ diagonal orthogonal matrices. So, $\phi(I) \in \mathcal{S}$. By Corollary 2.2, $(\phi(A), \phi(B))_{\xi}=(A, B)_{\xi}$. Since

$$
(\phi(I), \phi(I))_{\xi}=(I, I)_{\xi}=n+2 \alpha n^{2}+\alpha^{2} n^{3},
$$

we see that $\phi(I)=I$ by Proposition 2.3 Hence condition (1) holds.

To prove (2), note that $X \in V_{n}(\mathbf{F})$ satisfies

$$
0=(X, I)_{\xi}=(\alpha(\operatorname{tr} X) I+X, \alpha n I+I)=(\alpha n+1)^{2} \operatorname{tr} X
$$

if and only if $\operatorname{tr} X=0($ as $\alpha>-1 / n)$. Thus, if $X \in V_{n}(\mathbf{F})$ satisfies $\operatorname{tr} X=0$, then, by (1),

$$
(\phi(X), I)_{\xi}=(\phi(X), \phi(I))_{\xi}=(X, I)_{\xi}=0
$$

implies that $\operatorname{tr} \phi(X)=0$. Consequently, for any $X \in V_{n}(\mathbf{F})$, we have

$$
\operatorname{tr} \phi(X)=\operatorname{tr} \phi((\operatorname{tr} X) I / n)+\operatorname{tr} \phi(X-(\operatorname{tr} I) / n)=\operatorname{tr}[(\operatorname{tr} X) I / n]=\operatorname{tr} X .
$$

Now, suppose $A, B \in V_{n}(\mathbf{F})$. Then

$$
\begin{aligned}
(A, B) & +2 \alpha(\operatorname{tr} A)(\operatorname{tr} B)+\alpha^{2} n(\operatorname{tr} A)(\operatorname{tr} B) \\
= & (A+\alpha(\operatorname{tr} A) I, B+\alpha(\operatorname{tr} B) I) \\
= & (\xi(A), \xi(B))=(A, B)_{\xi} \\
= & (\phi(A), \phi(B))_{\xi}=(\xi \phi(A), \xi \phi(B)) \\
= & (\phi(A)+\alpha(\operatorname{tr} \phi(A)) I, \phi(B)+\alpha(\operatorname{tr} \phi(B)) I) \\
= & (\phi(A), \phi(B))+2 \alpha(\operatorname{tr} \phi(A))(\operatorname{tr} \phi(B))+\alpha^{2} n(\operatorname{tr} \phi(A))(\operatorname{tr} \phi(B)) \\
= & (\phi(A), \phi(B))+2 \alpha(\operatorname{tr} A)(\operatorname{tr} B)+\alpha^{2} n(\operatorname{tr} A)(\operatorname{tr} B) .
\end{aligned}
$$

Thus, condition (2) holds. 


\section{Proof OF THE MAIN THEOREM}

Using the results in the last section, we are ready to present the proof of Theorem 1.1 in the following. We shall often use the fact that $A \in \mathcal{R}$ if and only if $A=x x^{*}$ for some $x=\left(x_{1}, \ldots, x_{n}\right)^{t} \in \mathbf{F}^{n}$ with $\left|x_{j}\right|=1$ for all $j$.

The implication (c) $\Rightarrow$ (a) is clear. By Proposition 2.4 if (a) holds then (b) follows.

Now, suppose (b) holds, i.e., $\phi(\mathcal{R})=\mathcal{R}$. Then $\phi(E)=x x^{*}$ for some $x=$ $\left(x_{1}, \ldots, x_{n}\right)^{t} \in \mathbf{F}^{n}$ with $\left|x_{j}\right|=1$ for all $j$. We may replace $\phi$ by the operator defined by $A \mapsto X^{*} \phi(A) X$, where $X=\operatorname{diag}\left(x_{1}, \ldots, x_{n}\right)$, and therefore assume that

(3) $\phi(E)=E$.

We first consider the real case.

Lemma 3.1. Suppose $\phi$ is a linear operator on $V_{n}(\mathbf{R})$ satisfying $\phi(\mathcal{R})=\mathcal{R}$ and $\phi(E)=E$. Then there is a permutation matrix $P$ such that condition (c.i) in Theorem 1.1 holds.

Proof. Suppose $\phi$ satisfies the hypothesis. Let $f_{j}=e-2 e_{j}$ and $F_{j}=f_{j} f_{j}^{t}$ for $j=$ $1, \ldots, n$. Then $A=x x^{t} \in \mathcal{R}$, such that $x=\left(x_{1}, \ldots, x_{n}\right)^{t} \in \mathbf{R}^{n}$ with $x_{j} \in\{1,-1\}$ for all $j$, satisfies

$$
(A, E)=\left(x x^{t}, e e^{t}\right)=\left|\left(e^{t} x\right)\right|^{2}=(n-2)^{2}
$$

if and only if $A=F_{j}$ for some $j$. Hence, $\phi$ maps the set $\left\{F_{j}: 1 \leq j \leq n\right\}$ onto itself. We may assume that $\phi\left(F_{j}\right)=F_{j}$; otherwise, replace $\phi$ by a mapping of the form $A \mapsto P \phi(A) P^{t}$ for a suitable permutation matrix $P$. We shall show that this modified mapping $\phi$ is the identity operator on $V_{n}(\mathbf{R})$.

If $n \leq 3$, then $\phi(X)=X$ for all $X$ in the spanning set $\left\{I, F_{1}, \ldots, F_{n}\right\}$ of $V_{n}(\mathbf{R})$. Thus, $\phi$ is the identity operator, as asserted.

Suppose $n \geq 4$. There are matrices in $\mathcal{R}$ of the form $F_{j k}=x x^{t}$ with $x=$ $e-2\left(e_{j}+e_{k}\right)$ for $1 \leq j<k \leq n$. Note that $F_{j k}$ is the only matrix in $\mathcal{R}$ such that $\left(F_{j k}, E\right)=(n-4)^{2},\left(F_{j k}, F_{j}\right)=\left(F_{j k}, F_{k}\right)=(n-2)^{2}$, and $\left(F_{j k}, F_{r}\right)=(n-6)^{2}$ for all other $r \neq j, k$. Since

$$
\left(\phi\left(F_{j k}\right), X\right)=\left(\phi\left(F_{j k}\right), \phi(X)\right)=\left(F_{j k}, X\right)
$$

for any $X \in\left\{E, F_{1}, \ldots, F_{n}\right\}$, we see that $\phi\left(F_{j k}\right)=F_{j k}$ for all $j \neq k$. Observe that

$$
2\left(E_{j k}+E_{k j}\right)=E+F_{j k}-F_{j}-F_{k}
$$

and hence

$$
S=\left\{I, F_{1}, \ldots, F_{n}\right\} \cup\left\{F_{j k}: 1 \leq j<k \leq n\right\}
$$

is a spanning set of $V_{n}(\mathbf{R})$. Since $\phi$ fixes every element in this spanning set, it is the identity map on $V_{n}(\mathbf{R})$, as asserted.

Next, we turn to the complex case. The proof is more involved and is divided into several lemmas.

Since every complex rank one correlation matrix has the form $x x^{*}$ with $x=$ $\left(x_{1}, \ldots, x_{n}\right)^{t} \in \mathbf{C}^{n}$ satisfying $\left|x_{j}\right|=1$ for all $j$, the set $\mathcal{R}$ can be viewed as the orbit of the element $e e^{t}$ under the compact group of linear operators of the form 
$\psi_{P}$ defined by $A \mapsto P^{*} A P$ for any diagonal unitary matrix $P$. The tangent vectors of $\mathcal{R}$ at $E=e e^{t}$ can be computed by

$$
\left.\frac{d}{d s} \exp (i D(s)) e e^{t} \exp (-i D(s))\right|_{s=0}=i\left(D^{\prime}(0) e e^{t}-e e^{t} D^{\prime}(0)\right),
$$

where $D(s)$ is a smooth path of real diagonal matrices with $D(0)$ equal to the zero matrix. Thus, the tangent space of $\mathcal{R}$ at $e e^{t}$ is

$$
\begin{aligned}
\mathcal{T}_{E} & =\left\{i\left(D e e^{t}-e e^{t} D\right): D \text { is a real diagonal matrix }\right\} \\
& =\left\{i\left(x e^{t}-e x^{t}\right): x \in \mathbf{R}^{n}\right\}
\end{aligned}
$$

Since $\phi$ is a linear map satisfying $\phi(\mathcal{R})=\mathcal{R}$ and $\phi(E)=E$, we have $\phi\left(\mathcal{T}_{E}\right)=\mathcal{T}_{E}$.

Let $\mathcal{T}_{E}^{\perp}$ denote the orthogonal complement of $\mathcal{T}_{E}$ in $V_{n}(\mathbf{C})$ with respect to the inner product $(A, B)=\operatorname{tr}(A B)$ on $V_{n}(\mathbf{C})$. For each matrix in $V_{n}(\mathbf{C})$, write it as $X+i Y$ with $X$ real symmetric and $Y$ real skew-symmetric. Note that for $X \in M_{n}(\mathbf{F}), x, y \in \mathbf{F}^{n}$, we have $\operatorname{tr}\left(X y x^{t}\right)=x^{t} X y$. Thus $X+i Y \in \mathcal{T}_{E}^{\perp}$ if and only if for any $x \in \mathbf{R}^{n}$ we have

$$
\begin{aligned}
0 & =i\left(X+i Y, x e^{t}-e x^{t}\right)=\left(Y, e x^{t}-x e^{t}\right) \\
& =x^{t} Y e-e^{t} Y x=x^{t} Y e-x^{t} Y^{t} e=2 x^{t} Y e,
\end{aligned}
$$

i.e., $Y e=0$. Hence,

$$
\begin{aligned}
\mathcal{T}_{E}^{\perp}=\left\{X+i Y \in V_{n}(\mathbf{C}): X\right. & \text { is real symmetric and } \\
Y & \text { is real skew-symmetric satisfying } Y e=0\} .
\end{aligned}
$$

Since $\phi$ preserves $(\cdot, \cdot)$, we have $\phi\left(\mathcal{T}_{E}^{\perp}\right)=\mathcal{T}_{E}^{\perp}$.

Using a similar argument, we can show that for any complex rank one correlation matrix $Z=v v^{*}$, where $v \in \mathbf{C}^{n}$ with entries having moduli one, the tangent space of $\mathcal{R}$ at $Z$ is

$$
\begin{aligned}
\mathcal{T}_{Z} & =\left\{i\left(D v v^{*}-v v^{*} D\right): D \text { is a real diagonal matrix }\right\} \\
& =\left\{i V\left(D e e^{t}-e e^{t} D\right) V^{*}: D \text { is a real diagonal matrix }\right\} \\
& =V \mathcal{T}_{E} V^{*}
\end{aligned}
$$

where $V$ is the diagonal unitary matrix whose diagonal entries are the entries of $v$. Furthermore, one can show that the orthogonal complement of $\mathcal{T}_{Z}$ is

$$
\mathcal{T}_{Z}^{\perp}=V \mathcal{T}_{E}^{\perp} V^{*} .
$$

With this background, we can prove

Lemma 3.2. Let $Z \in \mathcal{R}$. Then $Z$ is a real correlation matrix if and only if $E \in$ $\mathcal{T}_{Z}^{\perp}$.

Proof. Suppose $Z \in \mathcal{R}$ is real. Then

$$
\mathcal{T}_{Z}=\{i(D Z-Z D): D \text { is a real diagonal matrix }\},
$$

and clearly $E \in \mathcal{T}_{Z}^{\perp}$.

Conversely, if $E \in T_{Z}^{\perp}$, then

$$
E=V^{*}(X+i Y) V
$$

where $X$ is real symmetric and $Y$ is real skew-symmetric such that $Y e=0$. Let $v=v_{1}+i v_{2}$, with $v_{1}, v_{2}$ real vectors. If $v_{1}$ or $v_{2}$ is zero, then $v v^{*}$ is real and we are done. If not, we will show that $v_{1}$ is a multiple of $v_{2}$. Then $v=\mu v_{2}$ for some 
complex unit $\mu$. It will then follows that $Z=v_{2} v_{2}^{t}$ is real. To achieve our goal, observe that

$$
V E V^{*}=X+i Y, \quad \text { i.e., } \quad\left(v_{1}+i v_{2}\right)\left(v_{1}-i v_{2}\right)^{t}=X+i Y,
$$

and hence

$$
Y=\left(v_{2} v_{1}^{t}-v_{1} v_{2}^{t}\right)
$$

Since $Y e=0$, we see that $v_{1}\left(v_{2}^{t} e\right)=v_{2}\left(v_{1}^{t} e\right)$. So, $v_{1}$ is a multiple of $v_{2}$, as asserted.

By Lemma 3.2 we can prove

Lemma 3.3. Suppose $\phi$ is a linear operator on $V_{n}(\mathbf{C})$ satisfying $\phi(\mathcal{R})=\mathcal{R}$ and $\phi(E)=E$. Then there exists a permutation matrix $P$ such that the mapping $\phi(A)=$ $P^{t} A P$ for all real matrices $A \in V_{n}(\mathbf{C})$.

Proof. Suppose $A$ is a real rank one correlation matrix and $\phi(A)=B$. Then $\phi\left(\mathcal{T}_{A}\right)=\mathcal{T}_{B}$. Since $\phi$ preserves the inner product $(\cdot, \cdot)$, we have $\phi\left(\mathcal{T}_{A}^{\perp}\right)=\mathcal{T}_{B}^{\perp}$. By the fact that $E \in \mathcal{T}_{A}^{\perp}$, we have $E=\phi(E) \in \phi\left(\mathcal{T}_{A}^{\perp}\right)=\mathcal{T}_{B}^{\perp}$, and hence $B$ is a real rank one correlation matrix.

Now, $\phi$ maps the set of real rank one correlation matrices into, and hence onto, itself. The restriction of $\phi$ on $V_{n}(\mathbf{R})$ is a real linear map satisfying $\phi(E)=E$ and $\phi\left(C_{n}(\mathbf{R})\right)=C_{n}(\mathbf{R})$. By Lemma 3.1, there exists a permutation matrix $P$ such that $\phi(A)=P^{t} A P$ for all matrices $A \in V_{n}(\mathbf{R})$, i.e., all real matrices in $V_{n}(\mathbf{C})$.

Let $P$ be the permutation matrix satisfying Lemma 3.3 . We may replace $\phi$ by the linear operator $A \mapsto P \phi(A) P^{t}$ and assume that

(4) $\phi(X)=X$ for all real matrices in $V_{n}(\mathbf{C})$.

Furthermore, observe that the set of real matrices in $V_{n}(\mathbf{C})$ is just the set $V_{n}(\mathbf{R})$, and a matrix $Z \in V_{n}(\mathbf{C})$ satisfies $(Z, X)=0$ for all $X \in V_{n}(\mathbf{R})$ if and only if $Z=i Y$ for some real skew-symmetric matrix $Y$. Since $\phi$ preserves the inner product $(\cdot, \cdot)$, we have

(5) If $Y$ is a real skew-symmetric matrix, then $\phi(i Y)=i \tilde{Y}$ for some real skewsymmetric matrix $\tilde{Y}$.

We complete the proof of our main theorem by establishing the following.

Lemma 3.4. Let $\mathcal{C}=\left\{i\left(E_{j k}-E_{k j}\right): 1 \leq j<k \leq n\right\}$. If $\phi$ is a linear operator on $V_{n}(\mathbf{C})$ satisfying $\phi(\mathcal{R})=\mathcal{R}$, and conditions $(3)-(5)$, then either

(i) $\phi(X)=X$ for all $X \in \mathcal{C}$, and hence Theorem 1.1 (c.i) holds; or

(ii) $\phi(X)=-X=X^{t}$ for all $X \in \mathcal{C}$, and hence Theorem 1.1 (c.ii) holds.

Proof. Let

$$
A=\left(i e_{1}+\sum_{j=2}^{n} e_{j}\right)\left(-i e_{1}+\sum_{j=2}^{n} e_{j}\right)^{t}=X+i Y \in \mathcal{R},
$$

where $X$ and $Y$ are real matrices. By (4), (5) and the fact that $\phi(\mathcal{R})=\mathcal{R}$, we see that $\phi(A)=x x^{*}=X+i \tilde{Y}$ for some real skew-symmetric matrix $\tilde{Y}$ and $x=\left(x_{1}, \ldots, x_{n}\right)^{t} \in \mathbf{C}^{n}$ with $\left|x_{j}\right|=1$ for all $j$. Comparing the real entries in $x x^{*}$ and $X+i \tilde{Y}$, we see that $x$ can be replaced by $x / x_{n}$ so that $x_{2}=\cdots=x_{n}=1$ and $x_{1} \in\{i,-i\}$. Thus, $\phi(Y)=Y$ or $-Y$. If $n=2$, then $\mathcal{C}=\{Y\}$ and we are done. Suppose $n \geq 3$ and the result is valid for linear maps on $V_{n-1}(\mathbf{C})$. We finish the 
proof by induction. We may assume that

(6) $\phi(A)=A$.

Otherwise, replace $\phi$ by the mapping $Z \mapsto \phi\left(Z^{t}\right)$. We will show that (i) holds.

For each $j=2, \ldots, n$,

$$
B_{j}=\left(i e_{1}-e_{j}+\sum_{p \neq 1, j} e_{p}\right)\left(-i e_{1}-e_{j}+\sum_{p \neq 1, j} e_{p}\right)^{t}=X_{j}+i Y_{j} \in \mathcal{R},
$$

where $X_{j}$ and $Y_{j}$ are real matrices. Similar to the analysis of $\phi(A)$, we can use (4), (5), and the fact that $\phi(\mathcal{R})=\mathcal{R}$ to conclude that $\phi\left(Y_{j}\right)=Y_{j}$ or $-Y_{j}$. Hence, $\phi$ maps the set $R=\left\{B_{2}, \ldots, B_{n}\right\} \cup\left\{B_{2}^{t}, \ldots, B_{n}^{t}\right\}$ onto itself.

Observe that

$$
S=\operatorname{span}\left\{i\left(E_{j k}-E_{k j}\right): 2 \leq j<k \leq n\right\}
$$

equals $\left(V_{n}(\mathbf{R}) \cup R\right)^{\perp}$. Since $\phi$ preserves the inner product by Proposition 2.5, one sees that $\phi(S)=S$. If $[a] \oplus Z=X+i Y \in V_{n}(\mathbf{C})$, where $X$ and $Y$ are real matrices, then $i Y \in S$. Thus, $\phi(Z)=X+i \tilde{Y}$ with $i \tilde{Y} \in S$, and $\phi(Z)$ must be of the form $[a] \oplus \tilde{Z}$. Now, one can apply the induction assumption to the linear operator $\psi$ on $V_{n-1}(\mathbf{C})$ defined by $\phi([a] \oplus Z)=[a] \oplus \psi(Z)$ to conclude that

(i') $\psi(Z)=Z$ for all $Z \in V_{n-1}(\mathbf{C})$, or

(ii') $\psi(Z)=\bar{Z}=Z^{t}$ for all $Z \in V_{n-1}(\mathbf{C})$.

It will be shown that (ii') does not hold. We first prove that

(7) $\phi\left(i\left(E_{1 j}-E_{j 1}\right)\right)=i\left(E_{1 j}-E_{j 1}\right)$ for $j=2, \ldots, n$.

If $n \geq 4$, then

$$
\left(\phi(A), \phi\left(B_{j}\right)\right)=\left(A, B_{j}\right)
$$

and we see that $\phi\left(B_{j}\right)=B_{j}$. Note that $i\left(E_{1 j}-E_{j 1}\right)=A-B_{j}+Y$ for some real matrix $Y$, and $\phi$ fixes $A, B_{j}$ and $Y$. Thus, $\phi$ fixes $i\left(E_{1 j}-E_{j 1}\right)$ as well.

If $n=3$, and (7) does not hold, then $\phi\left(B_{j}\right)=B_{j}^{t}$ for $j=2$ or 3 . Note that $B_{j}^{t}=B_{k}$ with $j+k=5$. So,

$$
\phi\left(2 I+2 i\left(E_{1 k}-E_{k 1}\right)\right)=\phi\left(A+B_{j}\right)=A+B_{k}=2 I+2 i\left(E_{1 j}-E_{j 1}\right) .
$$

Since $\phi(I)=I$, we see that

$$
\phi\left(i\left(E_{1 k}-E_{k 1}\right)\right)=i\left(E_{1 j}-E_{j 1}\right) .
$$

But then for $X=\left(e-(1+i) e_{k}\right)\left(e-(1-i) e_{k}\right)^{t} \in \mathcal{R}$, since $\phi\left(i E_{23}-E_{32}\right)=$ $\pm i\left(E_{23}-E_{32}\right)$ by $\left(\mathrm{i}^{\prime}\right)$ or $\left(\mathrm{ii}^{\prime}\right)$, we have

$$
\phi(X)=I+E_{1 j}+E_{j 1}+i\left(E_{1 j}-E_{j 1}\right) \pm i\left(E_{23}-E_{32}\right) \notin \mathcal{R}
$$

contradicting the fact that $\phi(\mathcal{R})=\mathcal{R}$. Hence, (7) also holds when $n=3$.

We are ready to prove that $\left(\right.$ ii $\left.^{\prime}\right)$ does not hold under the additional assumptions (6) and (7). If (ii') does hold, consider

$$
Z=\left(\sum_{j=1}^{n-1} e_{j}-i e_{n}\right)\left(\sum_{j=1}^{n-1} e_{j}+i e_{n}\right)^{t} \in \mathcal{R}
$$

Then by (4), (7) and (ii'), we see that

$$
\phi(Z)=\sum_{1 \leq j, k \leq n-1} E_{j k}+i\left(E_{1 n}-E_{n 1}\right)-\sum_{j=2}^{n-1} i\left(E_{j n}-E_{n j}\right) \notin \mathcal{R},
$$

contradicting the fact that $\phi(\mathcal{R})=\mathcal{R}$. Hence (i') holds, and (i) follows. 


\section{REFERENCES}

1. H. Auerbach, Sur les groupes bornés de substitutions, linéaires, C.R. Acad. Sci. Paris 195 (1932), 1367-1369.

2. M.D. Choi, Positive linear maps, Proceedings of Symposia in Pure Math., Amer. Math. Soc. 38 (2) (1982), 583-590.

3. C. Chevalley, Theory of Lie groups, Princeton University Press, Princeton, 1946. MR 7:412c

4. E. Deutsch and H. Schneider, Bounded groups and norm-hermitian matrices, Linear Algebra Appl. 9 (1974), 9-27. MR 52:3200

5. S. Pierce et. al., A Survey on Linear Preserver Problems, Linear and Multilinear Algebra 33 (1992), 1-129. MR 96c:15043

Department of Mathematics, The College of William and Mary, Williamsburg, VirGINIA 23187

E-mail address: ckli@math.wm.edu

Department of Mathematical Sciences, San Diego State University, San Diego, CalIFORNIA 92182

E-mail address: pierce@math.sdsu.edu 